\title{
Preoperative frailty is associated with progression of postoperative cardiac rehabilitation in patients undergoing cardiovascular surgery
}

\author{
Yasuhiro Arai ${ }^{1} \cdot$ Toru Kimura $^{1} \cdot$ Yuki Takahashi ${ }^{1} \cdot$ Takashi Hashimoto $^{1} \cdot$ Mamoru Arakawa $^{2} \cdot$ Homare Okamura $^{2}(\mathbb{D}$
}

Received: 23 November 2018 / Accepted: 1 April 2019 / Published online: 5 April 2019

(c) The Japanese Association for Thoracic Surgery 2019

\begin{abstract}
Objective Preoperative frailty affects the progression of cardiac rehabilitation (CR) after cardiovascular surgery. Different frailty assessment measures are available. However, it remains unclear which tool most likely predicts the progress of $\mathrm{CR}$. Our aim was to evaluate preoperative frailty using different methods and to identify the predictors in the progress of postoperative CR.

Methods Eighty-nine patients underwent elective cardiovascular surgery at our institution between May 2016 and April 2018. Mortality cases and patients without evaluation of preoperative frailty were excluded. This study included the remaining 78 patients. We divided the patients into two groups: 47 patients who achieved $100 \mathrm{~m}$ walking within 7 days after surgery (successful CR group) and 31 patients who achieved $100 \mathrm{~m}$ walking later than 8 days after surgery (delayed CR group). Preoperative frailty was assessed using the Kaigo-Yobo Check-List, Cardiovascular Health Study, Short Physical Performance Battery, and Clinical Frailty Scale.

Results The prevalence of frailty defined by these four measures was higher in the delayed CR group. The delayed CR group had lower nutritional status, serum hemoglobin level, serum albumin level, and psoas muscle index. Multivariable analysis demonstrated the Kaigo-Yobo Check-List score as an independent predictor for delayed CR (odds ratio 1.53, 95\% confidence interval 1.18-1.98, $p=0.001$ ) and Clinical Frailty Scale as an independent predictor for discharge to a health care facility (odds ratio 3.70, 95\% confidence interval 1.30-10.51, $p=0.014$ ).

Conclusions Among the various tools for assessing frailty, the Kaigo-Yobo Check-List was most likely to predict the progress of postoperative $\mathrm{CR}$ after elective cardiovascular surgery.
\end{abstract}

Keywords Frailty $\cdot$ Kaigo-Yobo Check-List · Cardiovascular surgery $\cdot$ Cardiac rehabilitation $\cdot 100 \mathrm{~m}$ walking

\section{Introduction}

Frailty is a geriatric syndrome reflecting increased vulnerability to stressors due to impairments in multiple physiologic systems. In the aging society, frailty has been a growing issue in patients undergoing surgery. Reportedly, frailty is a risk for postoperative complications and an independent predictor of in-hospital mortality, discharge to an institution, and decreased mid-term survival rate after cardiovascular surgery [1].

Homare Okamura

homareo@jadecom.jp

1 Department of Rehabilitation, Nerima Hikarigaoka Hospital, Tokyo, Japan

2 Department of Cardiovascular Surgery, Nerima Hikarigaoka Hospital, 2-11-1 Hikarigaoka, Nerima-Ku, Tokyo, Japan
Frailty comprises of various clinical phenotypes involving nutritional status, physical function, exhaustion, wasting, and inactivity. Preoperative malnutrition has been shown to be associated with increased morbidity, lower survival rate, prolonged hospital stay, and delayed cardiac rehabilitation (CR) progression after cardiovascular surgery [2,3]. Ogawa et al. showed that preoperative nutritional status was associated with the number of days to achieve independent walking after cardiac surgery [4]. In connection to frailty, sarcopenia is the age-associated decline in skeletal muscle mass and function, which represents a powerful predictor of mortality in the aging population [5, 6]. Among the several methods that evaluate sarcopenia, the psoas muscle area (PMA) is an objective and validated measure of muscle mass, which is measured using computed tomography (CT) [7]. Sarcopenia based on PMA is associated with higher mortality in various specialties, including trans-catheter aortic valve implantation (TAVI) [8]. 
However, the impact of preoperative frailty on the progress of postoperative CR remains unclear. Furthermore, a variety of frailty assessment tools are currently available, and it is difficult to determine which assessment tool should be used to predict the progress of postoperative CR. In this study, we aimed to assess preoperative frailty using several scales and identify the predictors for the progression of postoperative $\mathrm{CR}$ in patients undergoing cardiovascular surgery.

\section{Methods}

This was a retrospective study based on our institutional database. A total of 89 patients underwent elective cardiovascular surgery at our institution between May 2016 and April 2018. To evaluate the relationship between preoperative frailty and postoperative CR, two in-hospital mortality cases and nine patients who were not preoperatively evaluated for frailty were excluded (in-hospital mortality was $2.4 \%$ in this period). Thus, this study included the remaining 78 patients (44 males, 34 females, average age $71.7 \pm 11.8$ years).

The Japanese guideline for CR indicates that it is favorable to achieve $100 \mathrm{~m}$ walking within 7 days after surgery [9]. In order to identify the risk factors for the delayed progress of CR, we divided the patients into two groups based on the progress of CR: 47 patients who achieved $100 \mathrm{~m}$ walking day within 7 days after surgery (successful CR group) and 31 patients who achieved $100 \mathrm{~m}$ walking later than 8 days or were unable to achieve $100 \mathrm{~m}$ walking (delayed CR group). The Ethical Review Board of Nerima Hikarigaoka Hospital approved this study. All patients had previously given their permission for the use of their medical records for research purposes.

Data on all variables evaluated before surgery were extracted from electronic medical records. Age, sex, body mass index (BMI), Geriatric Nutritional Risk Index (GNRI) [10], co-morbidities, laboratory data, left ventricular ejection fraction (LVEF), preoperative co-morbidities, the psoas muscle area index (PMI, $\mathrm{cm}^{2} / \mathrm{m}^{2}$ ), and frailty status were measured before surgery.

GNRI was used to assess the preoperative nutritional status of the patients. GNRI was calculated as follows [10]: prior to surgery. PMI was calculated by standardizing PMA for height $\left(\mathrm{cm}^{2} / \mathrm{m}^{2}\right)$.

Frailty status was evaluated using three assessment scales including the Kaigo-Yobo Check-List [11], the Cardiovascular Health Study (CHS) [12], the Short Physical Performance Battery (SPPB) [13], and the Clinical Frailty Scale (CFS) [14]. These scales were measured the day before surgery. In accordance with the previous studies, the cut-off values of frailty in each scale were four points or more for the Kaigo-Yobo Check-List, three points or more for $\mathrm{CHS}$, and 8 points or less for SPPB [11-13].

The postoperative rehabilitation program at our hospital was based on the Guidelines for Rehabilitation in Patients with Cardiovascular Disease [9]. Except for the critically ill patients, postoperative rehabilitation started from the day after the surgery. The CR program was appropriately designed and modified according to an individual's body functions and general condition. The patients with the following symptoms did not move to the next level of exercise training in this study: (1) chest pain, severe shortness of breath, severe fatigue (Borg scale of $>13$ ), dizziness, lightheadedness, or leg pain; (2) cyanosis, facial pallor, or cold sweats; (3) tachypnea (>30/min); (4) atrial fibrillation or an increase in the incidence of arrhythmias; (5) ischemic ECG changes; (6) excessive changes in blood pressure; (7) an increase in heart rate by $>30$ beats per minute, or (8) a decrease in oxygen saturation to $<90 \%$. The progress in CR including $100 \mathrm{~m}$ walking was assessed. We did not apply the rehabilitation discontinuation criteria based on days after surgery. We comprehensively evaluated the patient's overall condition and consulted with doctors to determine whether postoperative rehabilitation should be carried out or not.

The data in this study were expressed as mean \pm standard deviation for continuous variables and as frequencies and percentages for categorical variables. Patient characteristics between groups were compared using the Fisher's exact test for categorical variables and the unpaired Student's $t$ test for continuous variables. Acute kidney injury was defined based on the Kidney Disease Improving Global Outcomes (KDIGO) clinical practice guidelines, i.e. the increase in

GNRI $=14.89 \times$ preoperative serum albumin $(\mathrm{g} / \mathrm{dL})$

$+[41.7 \times$ preoperative body weight $(\mathrm{kg}) /$ ideal body weight $(\mathrm{kg})]$,

*Calculation of ideal body weight:

$$
\begin{aligned}
& \text { male }=[\text { height }(\mathrm{cm})-100-(\text { height }-150) / 4] \\
& \text { female }=[\text { height }(\mathrm{cm})-100-(\text { height }-150) / 2.5] .
\end{aligned}
$$

PMA, which is a validated measure of sarcopenia, was measured at the level of the third lumbar vertebrae using the most recent preoperative $\mathrm{CT}$ scans done within 3 months serum creatinine value by $0.3 \mathrm{mg} / \mathrm{dL}$ or higher from the preoperative value within $48 \mathrm{~h}$ after surgery [15]. Low cardiac output syndrome was defined as (1) requiring intra-aortic balloon pump/percutaneous cardiopulmonary support to be weaned from cardiopulmonary bypass and/or (2) the need for adrenaline or more than $5 \mu \mathrm{g} / \mathrm{kg} / \mathrm{min}$ of dopamine or dobutamine after correction of electrolytes and preload in 
the intensive care unit [16]. Multivariable logistic regression analysis with $p<0.20$ for inclusion and $p>0.25$ for exclusion from the models was performed using the stepwise method to identify the independent predictors of (1) delayed CR and (2) discharge to a health care facility. Preoperative and intra-operative variables with $p<0.20$ in the univariate analysis were subjected to multivariable analysis. All statistical analyses were performed using SPSS software version 25 (IBM, New York, USA). A $p$ value of $<0.05$ was considered statistically significant.

\section{Results}

Preoperative patient characteristics per group are shown in Table 1. The patients in the delayed CR group had lower GNRI, serum albumin, and PMI than those in the successful CR group. Preoperative frailty status per group is shown in Table 1 . The Kaigo-Yobo Check-List, CHS points and CFS were higher and the SPPB point was lower in the delayed CR group as compared to the successful CR group. The prevalence of frailty status as defined by the Kaigo-Yobo Check-List, CHS, and SPPB

Table 1 Preoperative patient characteristics and preoperative frailty assessment

\begin{tabular}{|c|c|c|c|c|}
\hline Variable & Total $(n=78)$ & $\begin{array}{l}\text { Successful CR group } \\
(n=47)\end{array}$ & $\begin{array}{l}\text { Delayed CR group } \\
(n=31)\end{array}$ & $p$ value \\
\hline Age, years & $71.7 \pm 11.8$ & $69.8 \pm 12.7$ & $74.7 \pm 9.8$ & 0.076 \\
\hline Male, $n(\%)$ & $44(56)$ & $27(57)$ & $17(55)$ & 1.000 \\
\hline Body mass index, $\mathrm{kg} / \mathrm{m}^{2}$ & $23.6 \pm 4.4$ & $23.4 \pm 4.2$ & $23.8 \pm 4.7$ & 0.701 \\
\hline Geriatric nutritional risk index & $95.2 \pm 9.1$ & $97.3 \pm 8.9$ & $92.1 \pm 8.5$ & 0.011 \\
\hline Hypertension, $n(\%)$ & $54(69)$ & $30(64)$ & $24(77)$ & 0.223 \\
\hline Diabetes, $n(\%)$ & $25(32)$ & $14(30)$ & $11(35)$ & 0.627 \\
\hline Dyslipidemia, $n(\%)$ & $30(38)$ & $19(40)$ & $11(35)$ & 0.813 \\
\hline Renal insufficiency, $n(\%)^{\mathrm{a}}$ & $18(23)$ & $8(17)$ & $10(32)$ & 0.170 \\
\hline Old myocardial infarction, $n(\%)$ & $18(23)$ & $12(26)$ & $6(19)$ & 0.592 \\
\hline Heart failure, $n(\%)$ & $21(27)$ & $11(23)$ & $10(32)$ & 0.440 \\
\hline COPD, $n(\%)$ & $13(17)$ & $7(15)$ & $6(19)$ & 0.758 \\
\hline History of stroke, $n(\%)$ & $10(13)$ & $5(11)$ & $5(16)$ & 0.507 \\
\hline History of leg fracture, $n(\%)$ & $9(12)$ & $5(11)$ & $4(13)$ & 1.000 \\
\hline Serum hemoglobin, g/dL & $12.7 \pm 2.0$ & $13.1 \pm 1.2$ & $12.2 \pm 2.0$ & 0.079 \\
\hline Serum creatinine, $\mathrm{mg} / \mathrm{dL}$ & $1.41 \pm 1.75$ & $1.14 \pm 1.21$ & $1.82 \pm 2.30$ & 0.136 \\
\hline Serum albumin, $\mathrm{g} / \mathrm{dL}$ & $3.7 \pm 0.6$ & $3.9 \pm 0.5$ & $3.5 \pm 0.5$ & 0.004 \\
\hline Brain natriuretic peptide, $\mathrm{pg} / \mathrm{mL}$ & $338 \pm 457$ & $312 \pm 398$ & $379 \pm 535$ & 0.549 \\
\hline C-reactive protein, $\mathrm{mg} / \mathrm{dL}$ & $0.7 \pm 1.0$ & $0.5 \pm 0.7$ & $0.9 \pm 1.3$ & 0.138 \\
\hline Left ventricular ejection fraction, $\%$ & $58.0 \pm 16.0$ & $59.0 \pm 14.7$ & $56.5 \pm 18.0$ & 0.499 \\
\hline Psoas muscle index, $\mathrm{cm}^{2} / \mathrm{m}^{2}$ & $4.63 \pm 1.38$ & $4.98 \pm 1.40$ & $4.26 \pm 1.27$ & 0.047 \\
\hline Kaigo-Yobo Check-List, point & $3.2 \pm 2.4$ & $2.4 \pm 1.7$ & $4.5 \pm 2.6$ & 0.000 \\
\hline Frailty defined by Kaigo-Yobo Check-List, $n(\%)$ & $33(42)$ & $14(30)$ & $19(61)$ & 0.010 \\
\hline Cardiovascular Health Study, point & $1.7 \pm 1.6$ & $1.2 \pm 1.2$ & $2.4 \pm 1.8$ & 0.003 \\
\hline Frailty defined by CHS, $n(\%)$ & $20(26)$ & $8(17)$ & $12(39)$ & 0.038 \\
\hline Short Physical Performance Battery, point & $9.7 \pm 3.2$ & $10.3 \pm 2.6$ & $8.7 \pm 3.8$ & 0.044 \\
\hline Balance test, point & $3.4 \pm 1.1$ & $3.6 \pm 0.9$ & $3.1 \pm 1.2$ & 0.051 \\
\hline Gait test, point & $3.2 \pm 1.3$ & $3.5 \pm 1.1$ & $2.9 \pm 1.5$ & 0.072 \\
\hline Chair stand test, point & $3.0 \pm 1.4$ & $3.2 \pm 1.2$ & $2.7 \pm 1.5$ & 0.112 \\
\hline Frailty defined by SPPB, $n(\%)$ & $19(24)$ & $8(17)$ & $11(35)$ & 0.104 \\
\hline Clinical Frailty Scale & $3.5 \pm 0.9$ & $3.3 \pm 0.7$ & $4.0 \pm 0.8$ & $<0.001$ \\
\hline Grip strength, $\mathrm{kg}$ & $23.5 \pm 9.1$ & $24.6 \pm 9.6$ & $21.8 \pm 8.3$ & 0.176 \\
\hline Gait speed, m/s & $0.97 \pm 0.30$ & $1.01 \pm 0.29$ & $0.90 \pm 0.32$ & 0.115 \\
\hline
\end{tabular}

Values are number of patients $(\%)$ or mean $\pm \mathrm{SD}$

$C R$ cardiac rehabilitation, $C O P D$ chronic obstructive pulmonary disease, $C H S$ Cardiovascular Health Study, SPPB Short Physical Performance Battery

${ }^{\text {a }}$ Serum creatinine $>1.5 \mathrm{mg} / \mathrm{dL}$ 
was higher in the delayed CR group. However, grip strength and gait speed were similar between the groups. As shown in Table 2, the type of surgery except for mitral valve replacement, cardiopulmonary bypass, and cross-clamp times did not differ between the groups.

The progress of $\mathrm{CR}$ and postoperative morbidities are shown in Table 3. Postoperative hospital and intensive care unit stays were significantly longer in the delayed CR group. Regarding the progress of CR, the start day of rehabilitation was similar. However, the duration to achieve sitting, standing, walking, and $100 \mathrm{~m}$ walking were longer in the delayed CR group than in the successful CR group. The percentage of patients who achieved $100 \mathrm{~m}$ walking was also lower in the delayed CR group. However, the rate of discharge to own home did not differ between the groups. As for morbidities, the delayed CR group had a higher incidence of prolonged ventilator use.

Multivariable analysis was performed to determine independent factors for (1) delayed CR and (2) discharge to a health care facility. The predictors of the delayed $\mathrm{CR}$ are

Table 2 Operative date

\begin{tabular}{lllll}
\hline Variable & Total $(n=78)$ & $\begin{array}{l}\text { Successful CR } \\
\text { group }(n=47)\end{array}$ & $\begin{array}{l}\text { Delayed CR } \\
\text { group }(n=31)\end{array}$ & $p$ value \\
\hline $\begin{array}{l}\text { Surgical procedure } \\
\text { CABG, } n(\%)\end{array}$ & $31(40)$ & $18(38)$ & $13(42)$ & 0.815 \\
Valve surgery, $n(\%)$ & $46(59)$ & $28(60)$ & $18(58)$ & 1.000 \\
$\quad$ Aortic valve replacement, $n(\%)$ & $27(35)$ & $17(36)$ & $10(32)$ & 0.636 \\
$\quad$ Mitral valve replacement, $n(\%)$ & $10(13)$ & $3(6)$ & $7(23)$ & 0.025 \\
$\quad$ Mitral valve repair, $n(\%)$ & $14(18)$ & $10(21)$ & $4(13)$ & 0.386 \\
CABG+ valvular procedure, $n(\%)$ & $7(9)$ & $3(6)$ & $4(13)$ & 0.427 \\
Aortic surgery, $n(\%)$ & $9(12)$ & $4(9)$ & $5(16)$ & 0.471 \\
$\quad$ Ascending aorta replacement, $n(\%)$ & $6(8)$ & $3(6)$ & $3(10)$ & 0.677 \\
$\quad$ Aortic arch replacement, $n(\%)$ & $6(8)$ & $3(6)$ & $3(10)$ & 0.677 \\
Other, $n(\%)$ & $2(3)$ & $2(4)$ & $0(0)$ & 0.515 \\
CPB time, min & $165 \pm 52$ & $159 \pm 52$ & $175 \pm 52$ & 0.249 \\
Cross-clamp time, min & $117 \pm 36$ & $114 \pm 33$ & $122 \pm 41$ & 0.415 \\
\hline
\end{tabular}

Values are number of patients $(\%)$ or mean \pm SD

$C R$ cardiac rehabilitation, $C A B G$ coronary artery bypass grafting, $C P B$ cardiopulmonary bypass

Table 3 Progress of cardiac rehabilitation

\begin{tabular}{|c|c|c|c|c|}
\hline Variable & Total $(n=78)$ & $\begin{array}{l}\text { Successful CR } \\
\text { group }(n=47)\end{array}$ & $\begin{array}{l}\text { Delayed CR } \\
\text { group }(n=31)\end{array}$ & $p$ value \\
\hline Intensive care unit stay, day & $4.3 \pm 1.7$ & $3.7 \pm 1.2$ & $5.3 \pm 1.9$ & $<0.001$ \\
\hline Postoperative hospital stay, day & $20.5 \pm 10.2$ & $16.3 \pm 6.2$ & $26.8 \pm 11.9$ & $<0.001$ \\
\hline \multicolumn{5}{|l|}{ Progress of rehabilitation } \\
\hline Rehabilitation start day, day & $1.3 \pm 1.0$ & $1.2 \pm 0.6$ & $1.6 \pm 1.4$ & 0.136 \\
\hline Sitting start day, day & $1.6 \pm 1.2$ & $1.3 \pm 0.8$ & $2.2 \pm 1.6$ & 0.007 \\
\hline Standing start day, day & $2.8 \pm 2.9$ & $2.0 \pm 1.3$ & $4.1 \pm 4.0$ & 0.006 \\
\hline Walking start day, day & $5.4 \pm 4.7$ & $4.0 \pm 1.4$ & $7.5 \pm 6.8$ & 0.001 \\
\hline $100 \mathrm{~m}$ walking day, day & $7.9 \pm 4.6$ & $5.9 \pm 1.2$ & $11.5 \pm 5.5$ & $<0.001$ \\
\hline Achievement of $100 \mathrm{~m}$ gait, $n(\%)$ & $74(95)$ & $47(100)$ & $27(87)$ & 0.022 \\
\hline Discharge to own home, $n(\%)$ & $69(88)$ & $43(91)$ & $26(84)$ & 0.471 \\
\hline \multicolumn{5}{|l|}{ Postoperative morbidity } \\
\hline Stroke, $n(\%)$ & $1(1)$ & $0(0)$ & $1(3)$ & 0.397 \\
\hline Ventilator usage time, min & $2031 \pm 2709$ & $1439 \pm 859$ & $2928 \pm 4039$ & 0.052 \\
\hline Prolonged ventilator use $>48 \mathrm{~h}, n(\%)$ & $9(12)$ & $1(2)$ & $8(26)$ & 0.002 \\
\hline Acute kidney injury, n (\%) & $6(8)$ & $2(4)$ & $4(13)$ & 0.208 \\
\hline Low cardiac output syndrome, $n(\%)$ & $7(9)$ & $2(4)$ & $5(16)$ & 0.107 \\
\hline
\end{tabular}

Values are number of patients $(\%)$ or mean $\pm \mathrm{SD}$

$C R$ cardiac rehabilitation 
shown in Table 4. The Kaigo-Yobo Check-List score was found to be an independent predictor for delayed CR (odds ratio $=1.53,95 \%$ confidence interval $1.18-1.98, p=0.001$ ). The predictors of discharge to a health care facility are shown in Table 5. Multivariable analysis revealed CFS as an independent predictor for delayed CR (odds ratio $=3.70$, 95\% confidence interval 1.30-10.51, $p=0.014)$.

\section{Discussion}

The principal finding in this study was that Kaigo-Yobo Check-List was independently associated with the delayed progression of CR after elective cardiovascular surgery among several of the frailty assessment tools. The prevalence of preoperative frailty and the incidence of postoperative prolonged ventilator use were higher in the delayed CR group than in the successful CR group.

Currently, various scales have been used for the assessment of frailty, which include CHS, Kihon Check-List [17] and gait speed, nutrition status, and SPPB. In general, there are two types of frailty assessment measures. One is a comprehensive frailty evaluation that incorporates several aspects of phenotypes. For example, CHS evaluated weight loss, grip strength, self-reported exhaustion, slowness, and low physical activity level to evaluate frailty. However, this type of assessment is cumbersome and time-consuming. The other is a single performance measure, such as gait speed and grip strength. The concern in using a single performance measure is that it is unclear whether a single index can represent the clinical impact of several phenotypes of frailty. No matter which scale is used, the assessment measure should
Table 4 Predictors of delayed cardiac rehabilitation

\begin{tabular}{|c|c|c|c|c|}
\hline \multirow[t]{2}{*}{ Variable } & \multicolumn{2}{|l|}{ Univariate } & \multicolumn{2}{|l|}{ Multivariable } \\
\hline & OR $(95 \% \mathrm{CI})$ & $p$ value & OR $(95 \%$ CI $)$ & $p$ value \\
\hline Age, years & $1.04(0.99-1.09)$ & 0.083 & - & - \\
\hline Male, $n(\%)$ & $0.89(0.36-2.24)$ & 0.820 & - & - \\
\hline Body mass index, $\mathrm{kg} / \mathrm{m}^{2}$ & $1.02(0.92-1.13)$ & 0.697 & - & - \\
\hline Geriatric nutritional risk index & $0.94(0.89-0.99)$ & 0.014 & - & - \\
\hline Hypertension, $n(\%)$ & $1.94(0.69-5.45)$ & 0.207 & - & - \\
\hline Diabetes, $n(\%)$ & $1.30(0.49-3.40)$ & 0.598 & - & - \\
\hline Dyslipidemia, $n(\%)$ & $0.81(0.32-2.07)$ & 0.661 & - & - \\
\hline Renal insufficiency $^{\mathrm{a}}, n(\%)$ & $2.32(0.80-6.77)$ & 0.123 & - & - \\
\hline Old myocardial infarction, $n(\%)$ & $0.70(0.23-2.12)$ & 0.527 & - & - \\
\hline Heart failure, $n(\%)$ & $1.56(0.57-4.29)$ & 0.390 & - & - \\
\hline COPD, $n(\%)$ & $1.37(0.41-4.55)$ & 0.606 & - & - \\
\hline Stroke, $n(\%)$ & $1.61(0.43-6.12)$ & 0.481 & - & - \\
\hline Fracture of legs, $n(\%)$ & $1.24(0.31-5.05)$ & 0.760 & - & - \\
\hline Serum hemoglobin, $\mathrm{g} / \mathrm{dL}$ & $0.81(0.63-1.03)$ & 0.083 & - & - \\
\hline Serum creatinine, $\mathrm{mg} / \mathrm{dL}$ & $1.26(0.95-1.68)$ & 0.113 & - & - \\
\hline Serum albumin, g/dL & $0.28(0.11-0.71)$ & 0.007 & $0.44(0.16-1.23)$ & 0.118 \\
\hline Brain natriuretic peptide, $\mathrm{pg} / \mathrm{mL}$ & $1.00(0.99-1.00)$ & 0.545 & - & - \\
\hline C-reactive protein, $\mathrm{mg} / \mathrm{dL}$ & $1.48(0.91-2.41)$ & 0.118 & $1.49(0.83-2.65)$ & 0.179 \\
\hline Left ventricular ejection fraction, $\%$ & $0.99(0.96-1.02)$ & 0.494 & - & - \\
\hline Psoas muscle index, $\mathrm{cm}^{2} / \mathrm{m}^{2}$ & $0.70(0.49-1.02)$ & 0.062 & - & - \\
\hline Kaigo-Yobo Check-List, point & $1.56(1.23-1.99)$ & $<0.001$ & $1.53(1.18-1.98)$ & 0.001 \\
\hline Cardiovascular Health Study, point & $1.67(1.20-2.32)$ & 0.002 & - & - \\
\hline Short Physical Performance Battery, point & $0.85(0.73-0.99)$ & 0.037 & - & - \\
\hline Clinical Frailty Scale, point & $3.11(1.62-6.00)$ & 0.001 & & \\
\hline Coronary artery bypass grafting, $n(\%)$ & $1.16(0.46-2.93)$ & 0.748 & - & - \\
\hline Valve surgery, $n(\%)$ & $0.94(0.37-2.36)$ & 0.894 & - & - \\
\hline Aortic surgery, $n(\%)$ & $2.07(0.51-8.40)$ & 0.310 & - & - \\
\hline CPB time, $\min$ & $1.01(0.99-1.02)$ & 0.246 & - & - \\
\hline Cross-clamp time, min & $1.01(0.99-1.02)$ & 0.409 & - & - \\
\hline
\end{tabular}

${ }^{\text {a }}$ Serum creatinine $>1.5 \mathrm{mg} / \mathrm{dL}$

$O R$ odds ratio, $C I$ confidence interval, $C O P D$ chronic obstructive pulmonary disease, $C P B$ cardiopulmonary bypass 
Table 5 Predictors of discharge to a health care facility

\begin{tabular}{|c|c|c|c|c|}
\hline \multirow[t]{2}{*}{ Variable } & \multicolumn{2}{|l|}{ Univariate } & \multicolumn{2}{|l|}{ Multivariable } \\
\hline & OR $(95 \% \mathrm{CI})$ & $p$ value & OR $(95 \% \mathrm{CI})$ & $p$ value \\
\hline Age, years & $1.12(1.01-1.24)$ & 0.035 & - & - \\
\hline Male, $n(\%)$ & $0.58(0.14-2.35)$ & 0.445 & - & - \\
\hline Body mass index, $\mathrm{kg} / \mathrm{m}^{2}$ & $1.04(0.89-1.21)$ & 0.656 & - & - \\
\hline Geriatric nutritional risk index & $0.94(0.87-1.01)$ & 0.100 & - & - \\
\hline Hypertension, $n(\%)$ & $1.64(0.31-8.54)$ & 0.558 & - & - \\
\hline Diabetes, $n(\%)$ & $0.57(0.11-2.97)$ & 0.506 & - & - \\
\hline Dyslipidemia, $n(\%)$ & $1.32(0.33-5.38)$ & 0.696 & - & - \\
\hline Renal insufficiency ${ }^{\mathrm{a}}, n(\%)$ & $1.80(0.40-8.06)$ & 0.442 & - & - \\
\hline Old myocardial infarction, $n(\%)$ & - & 0.998 & - & - \\
\hline Heart failure, $n(\%)$ & $1.42(0.32-6.26)$ & 0.646 & - & - \\
\hline COPD, $n(\%)$ & $2.95(0.63-13.75)$ & 0.168 & - & - \\
\hline Stroke, $n(\%)$ & $0.83(0.09-7.47)$ & 0.871 & - & - \\
\hline Fracture of legs, $n(\%)$ & $2.53(0.44-14.64)$ & 0.300 & - & - \\
\hline Serum hemoglobin, $\mathrm{g} / \mathrm{dL}$ & $0.83(0.58-1.19)$ & 0.310 & - & - \\
\hline Serum creatinine, $\mathrm{mg} / \mathrm{dL}$ & $1.27(0.95-1.70)$ & 0.102 & - & - \\
\hline Serum albumin, g/dL & $0.39(0.12-1.33)$ & 0.134 & - & - \\
\hline Brain natriuretic peptide, $\mathrm{pg} / \mathrm{mL}$ & $1.00(0.99-1.00)$ & 0.909 & - & - \\
\hline C-reactive protein, $\mathrm{mg} / \mathrm{dL}$ & $1.22(0.69-2.15)$ & 0.491 & - & - \\
\hline Left ventricular ejection fraction, $\%$ & $0.99(0.95-1.03)$ & 0.655 & - & - \\
\hline Psoas muscle index, $\mathrm{cm}^{2} / \mathrm{m}^{2}$ & $0.66(0.35-1.22)$ & 0.184 & - & - \\
\hline Kaigo-Yobo Check-List, point & $1.37(1.01-1.87)$ & 0.044 & - & - \\
\hline Cardiovascular Health Study, point & $1.34(0.88-2.04)$ & 0.172 & - & - \\
\hline Short Physical Performance Battery, point & $0.80(0.67-0.96)$ & 0.019 & - & - \\
\hline Clinical Frailty Scale, point & $3.91(1.48-10.37)$ & 0.006 & $3.70(1.30-10.51)$ & 0.014 \\
\hline Coronary artery bypass grafting, $n(\%)$ & $2.07(0.51-8.40)$ & 0.310 & - & - \\
\hline Valve surgery, $n(\%)$ & $0.16(0.03-0.842)$ & 0.030 & $0.27(0.04-1.80)$ & 0.176 \\
\hline Aortic surgery, $n(\%)$ & $10.24(2.07-50.67)$ & 0.004 & $5.57(0.73-42.38)$ & 0.097 \\
\hline CPB time, $\min$ & $1.02(1.00-1.03)$ & 0.055 & - & - \\
\hline Cross-clamp time, min & $0.99(0.97-1.02)$ & 0.609 & - & - \\
\hline
\end{tabular}

$O R$ odds ratio, $C I$ confidence interval, $C O P D$ chronic obstructive pulmonary disease, $C P B$ cardiopulmonary bypass

${ }^{\text {a }}$ Serum creatinine $>1.5 \mathrm{mg} / \mathrm{dL}$ be reproducible, easy to measure in clinical practice, and highly predictive for outcomes.

In this study, we evaluated frailty using four comprehensive assessment tools: Kaigo-Yobo Check-List, CHS, SPPB, and CFS. The Kaigo-Yobo Check-List is easy to use in a clinical setting and can be evaluated in a short time with a few questions (15 items). The Kaigo-Yobo CheckList comprises 15 easy-to-answer questions, which mainly focus on three components: being homebound, falling, and malnutrition [11]. Unlike the CHS advocated by Fried and colleagues [12], the Kaigo-Yobo Check-List consists of only question items and does not require evaluation of body functions such as walking speed. The three components included in the Kaigo-Yobo Check-List were shown to be correlated with five characteristics (shrinking, exhaustion, low activity, slowness, weakness) that Fried et al. proposed as a constitution of frailty $[12,18]$. Kojima et al. showed that the frailty status quantified by the Kaigo-Yobo Check-List was shown to be a predictor in the newly certified longterm care insurance and/or mortality over 3 years [19]. CHS is a general method of evaluating physical frailty that is used worldwide. SPPB is a method that evaluates the more detailed physical functions as compared to CHS; therefore, it was adopted as a method of evaluating frailty in this study. CFS is a subjective evaluation method that does not require expert knowledge and is a useful index to easily evaluate the prognosis of the elderly $[14,20]$. CFS has been widely used in the assessment of patients undergoing TAVI. Preoperative CFS is reportedly associated with the completion rate of postoperative CR [21].

In the recent years, the impact of sarcopenia on clinical outcomes has gained attention because sarcopenia overlaps 
with the concept of frailty. In most papers, psoas muscle measurement has widely been used for defining sarcopenia [7, 8]. Lower PMA has been shown to be associated with mortality in various specialties, including TAVI [8]. Nutritional status defined by GNRI was also reported to be associated with delayed CR [4]. However, neither PMI nor GNRI was independently associated with CR progression in this study. These findings suggest that comprehensive assessment of frailty, rather than the impairment in one of clinical manifestations of frailty such as loss of muscle mass and malnutrition, might be more helpful in predicting the progress of CR.

The delayed CR group had a higher incidence of prolonged ventilator use than the successful CR group. Previous studies have reported that prolonged ventilator use after cardiac surgery is related to the number of days from surgery until achievement of independent gait [22] and hospital days [23]. As age increases and physical function declines, respiratory function declines [24, 25]. Frail patients have systemic vulnerability to stressors and respiratory muscle function can also be impaired, leading to longer dependence on the ventilator postoperatively.

Not surprisingly, postoperative hospital stay was longer in the delayed CR group than in the successful CR group. However, the rates of discharge to own home were similar between the two groups. These results suggest that postoperative rehabilitation enable patients to be discharged to their own homes, even in those with frailty and delayed progress of CR.

There are several advantages in the preoperative prediction of delayed CR patients. First, it helps steer therapy toward surgical, trans-catheter, or medical treatment. In the current era, various treatment options are available for cardiovascular diseases and providing optimal treatment to each patient is increasingly needed. By predicting the progress of postoperative $\mathrm{CR}$, surgeons can have a more comprehensive assessment of their patient and provide valuable information for patient counseling. Second, patients with frailty, as defined by the Kaigo-Yobo Check-List, may be the optimal target population that can benefit from preoperative interventions that facilitate the progress of postoperative CR. Although evidence about the efficacy of preoperative intervention such as aggressive prehabilitation and nutritional supplementation is currently sparse, identification of frail patients can help find candidates for preoperative intervention. Miguelena et al. showed that quality of life can improve after cardiac surgery even in frail elderly patients [26].

\section{Study limitations}

There are several limitations to this study. First, this is a single-center study involving a small sample size. Second, patients in this study underwent various types of surgeries which can affect the recovery from surgery, although there was no difference in types of surgeries between groups. Third, the impact of the delayed progression of postoperative CR on the long-term outcomes is not clear. In this study, we only focused on the predictors of delayed acquisition of $100 \mathrm{~m}$ walking after surgery. It is important to compare the outcomes after discharge between the groups.

\section{Conclusion}

Preoperative Kaigo-Yobo Check-List was the independent predictor for the progression of postoperative $\mathrm{CR}$ in patients undergoing cardiovascular surgery. Preoperative frailty assessment using the Kaigo-Yobo Check-List may help identify patients who will benefit from interventions such as prehabilitation and nutritional supplementation.

\section{Compliance with ethical standards}

Conflict of interest The authors have declared that no conflict of interest exists.

\section{References}

1. Sundermann S, Dademasch A, Praetorius J, Kempfert J, Dewey $\mathrm{T}$, Falk V, et al. Comprehensive assessment of frailty for elderly high-risk patients undergoing cardiac surgery. Eur J Cardiothorac Surg. 2011;39:33-7.

2. Lomivorotov VV, Efremov SM, Boboshko VA, Nikolaev DA, Vedernikov PE, Deryagin MN, et al. Prognostic value of nutritional screening tools for patients scheduled cardiac surgery. Interact Cardiovasc Thorac Surg. 2013;16:612-8.

3. Engleman DT, Admas DH, Byrne JG, Aranki SF, Collins JJ Jr, Couper GS, et al. Impact of body mass index and albumin on morbidity and mortality after cardiac surgery. J Thorac Cardiovasc Surg. 1999;118:866-73.

4. Ogawa M, Izawa KP, Satomi-Kobayashi S, Kitamura A, Ono R, Sakai Y, et al. Poor preoperative nutritional status is an important predictor of the retardation of rehabilitation after cardiac surgery in elderly cardiac patients. Aging Clin Exp Res. 2017;29:283-90.

5. Rajabali N, Rolfson D, Bagshaw SM. Assessment and utility of frailty measures in critical illness, cardiology, and cardiac surgery. Can J Cardiol. 2016;32:1157-65.

6. Schopfer DW, Forman DE. Cardiac rehabilitation in older adults. Can J Cardiol. 2016;32:1088-96.

7. Hamaguchi Y, Kaido T, Okumura S, Kobayashi A, Hammad A, Tamai Y, et al. Proposal for new diagnostic criteria for low skeletal muscle mass based on computed tomography imaging in Asian adults. Nutrition. 2016;32:1200-5.

8. Garg L, Agrawal S, Pew T, Hanzel GS, Abbas AE, Gallagher MJ, et al. Psoas muscle area as a predictor of outcomes in trancatheter aortic valve implantation. Am J Cardiol. 2017;119:457-60.

9. JCS Joint Working Group. Guidelines for rehabilitation in patients with cardiovascular disease (JCS 2012). Circ J. 2014;78:2022-93.

10. Bouillanne O, Morineau G, Dupont C, Coulombel I, Vincent JP, Nicolis I, et al. Geriatric nutritional risk index: a new index 
for evaluating at-risk elderly medical patients. Am J Clin Nutr. 2005;82:777-83.

11. Shinkai S, Watanabe N, Yoshida H, Fujiwara Y, Amano H, Lee S, et al. Research on screening for frailty: development of "the KaigoYobo Checklist". Nihon Koshu Eisei Zasshi. 2010;57:345-54.

12. Fried LP, Tangen CM, Walston J, Newman AB, Hirsch C, Gottdiener J, et al. Frailty in older adults: evidence for a phenotype. J Gerontol A Biol Sci Med Sci. 2001;56:146-56.

13. Cruz-Jentoft AJ, Baeyens JP, Bauer JM, Boirie Y, Cederholm T, Landi F, et al. Sarcopenia: European consensus on definition and diagnosis: report of the European Working Group on sarcopenia in older people. Age Ageing. 2010;39:412-23.

14. Rockwood K, Song X, MacKnight C, Bergman H, Hogan DB, Mcdowell I, et al. A global clinical measure of fitness and frailty in elderly people. CMAJ. 2005;173:489-95.

15. Kidney Disease: Improving Global Outcomes (KDIGO) Acute Kidney Injury Work Group. KDIGO clinical practice guideline for acute kidney injury. Kidney Int Suppl. 2012;2:1-138.

16. Fukui T, Tabata M, Motira S, Takanashi S. Early and long-term outcomes of coronary artery bypass grafting in patients with acute coronary syndrome versus stable angina pectoris. J Thorac Cardiovasc Surg. 2013;145:1577-83.

17. Sewo Sampaio PY, Sampaio RA, Yamada M, Arai H. Systematic review of the Kihon Checklist: is it a reliable assessment of frailty? Geriatr Gerontol Int. 2016;16:893-902.

18. Shinkai S, Watanabe N, Yoshida H, Fujiwara Y, Nishi M, Fukaya T, et al. Validity of the "Kaigo-Yobo Check-List" as a frailty index. Nihon Koshu Eisei Zasshi. 2013;60:262-74.

19. Kojima G, Taniguchi Y, Kitamura A, Shinkai S. Are the Kihon Checklist and the Kaigo-Yobo Checklist compatible with the frailty index? J Am Med Dir Assoc. 2018;19:797-800.
20. Gregorevic KJ, Hubbard RE, Lim WK, Katz B. The clinical frailty scale predicts functional decline and mortality when used by junior medical staff: a prospective cohort study. BMC Geriatr. 2016;16:117.

21. Kimber DE, Kehler DS, Lytwyn J, Boreskie KF, Jung P, Alexander B, et al. Pre-operative frailty status is associated with cardiac rehabilitation completion: a retrospective cohort study. J Clin Med. 2018;7:560.

22. Cerri AP, Bellelli G, Massone A, Pittella F, Landi F, Zambon A, et al. Sarcopenia and malnutrition in acutely ill hospitalized elderly: prevalence and outcomes. Clin Nutr. 2015;34:745-51.

23. Oura K, Morisawa T, Kamisaka K, Saitoh M, Hanafusa M, Yuguchi S, et al. Determinants of prolonged mechanical ventilation after cardiac surgery. Kyobu Geka. 2014;67:528-32.

24. Giua R, Pedone C, Scarlata S, Carrozzo I, Rossi FF, Valiani V, et al. Relationship between respiratory muscle strength and physical performance in elderly hospitalization patients. Rejuvenation Res. 2014;17:366-71.

25. Buchman AS, Boyle PA, Wilson RS, Leurgans S, Shan RC, Bennett DA. Respiratory muscle strength predicts decline in mobility in older persons. Neuroepidemiology. 2008;31:174-80.

26. Miguelena J, Lopez-Menendez J, Prada PC, Rodriguez-Roda J, Martin M, Vigil-Escalera C, et al. Influence of preoperative frailty on health-related quality of life after cardiac surgery. Ann Thorac Surg. 2019.https://doi.org/10.1016/S0003-4965(19)30098-0

Publisher's Note Springer Nature remains neutral with regard to jurisdictional claims in published maps and institutional affiliations. 